hep-th/0406224

ITP-UH-20/04

\title{
Topological B-Model on Weighted Projective Spaces and Self-Dual Models in Four Dimensions
}

\author{
Alexander D. Popov* and Martin Wolf \\ Institut für Theoretische Physik \\ Universität Hannover \\ Appelstraße 2, 30167 Hannover, Germany \\ E-mail: popov, wolf@itp.uni-hannover.de
}

\begin{abstract}
It was recently shown by Witten on the basis of several examples that the topological $B$-model whose target space is a Calabi-Yau (CY) supermanifold is equivalent to holomorphic Chern-Simons (hCS) theory on the same supermanifold. Moreover, for the supertwistor space $\mathbb{C} P^{3 \mid 4}$ as target space, it has been demonstrated that hCS theory on $\mathbb{C} P^{3 \mid 4}$ is equivalent to self-dual $\mathcal{N}=4$ super Yang-Mills (SYM) theory in four dimensions. We consider as target spaces for the $B$-model the weighted projective spaces $W \mathbb{C} P^{3 \mid 2}(1,1,1,1 \mid p, q)$ with two fermionic coordinates of weight $p$ and $q$, respectively - which are CY supermanifolds for $p+q=4$ - and discuss hCS theory on them. By using twistor techniques, we obtain certain field theories in four dimensions which are equivalent to hCS theory. These theories turn out to be self-dual truncations of $\mathcal{N}=4$ SYM theory or of its twisted (topological) version.
\end{abstract}

June, 2004

* On leave from Laboratory of Theoretical Phyiscs, JINR, Dubna, Russia. 


\section{Introduction and results}

Recently, Witten has shown [1] that there is a one-to-one correspondence between the moduli spaces of holomorphic Chern-Simons (hCS) theory [2] on the Calabi-Yau (CY) supermanifold $\mathbb{C} P^{3 \mid 4} \backslash \mathbb{C} P^{1 \mid 4}$ and of self-dual $\mathcal{N}=4$ super Yang-Mills (SYM) theory on $\mathbb{R}^{4}$ with a metric of signature $(++++)$ or $(++--)$. It was also shown that hCS theory on $\mathbb{C} P^{3 \mid 4}$ is related with the $B$-type open topological string (the $B$-model) having $\mathbb{C} P^{3 \mid 4}$ as target space (for related works see [3] - [9]). Therefore, string theory considerations can help to clarify properties of quantum $\mathcal{N}=4$ SYM theory (see e.g. [10 - 113]).

Recall that the fermionic coordinates $\eta_{i}(i=1, \ldots, 4)$ of the supermanifold $\mathbb{C} P^{3 / 4}=$ $W \mathbb{C} P^{3 \mid 4}(1,1,1,1 \mid 1,1,1,1)$ take values in the holomorphic line bundle $\mathcal{O}(1)$. As the product $\prod_{i=1}^{4} \mathrm{~d} \eta_{i}$ takes values in $\mathcal{O}(-4)$ and the holomorphic measure on $\mathbb{C} P^{3}$ is $\mathcal{O}(4)$ valued, the full holomorphic measure on $\mathbb{C} P^{3 \mid 4}$ is globally defined and nowhere vanishing. Thus, the supertwistor space $\mathbb{C} P^{3 \mid 4}$ is a $\mathrm{CY}$ supermanifold [1]. In this note, we will focus on the weighted projective spaces $W \mathbb{C} P^{3 \mid 2}(1,1,1,1 \mid p, q)$ with $(p, q)$ equal to $(1,3),(2,2)$ and $(4,0)$. Since $p+q=4$ for these spaces, they are CY supermanifolds due to the above reasoning. We describe hCS theory on open subsets of these weighted projective spaces and derive (equivalent) field theories on $\mathbb{R}^{4}$ from it. We show that these models are related with different self-dual truncations of either $\mathcal{N}=4$ SYM theory or its twisted (topological) version.

\section{Twistor geometry}

Local coordinates. Our starting point is the complex projective space $\mathbb{C} P^{3}$ (twistor space) with homogeneous coordinates $\left(\omega^{\alpha}, \lambda_{\dot{\alpha}}\right)$. These coordinates are subject to the equivalence relation $\left(\omega^{\alpha}, \lambda_{\dot{\alpha}}\right) \sim\left(t \omega^{\alpha}, t \lambda_{\dot{\alpha}}\right)$ with $t \in \mathbb{C}^{*}$. Here and in the following, $\alpha, \beta, \ldots=1,2$ and $\dot{\alpha}, \dot{\beta}, \ldots=1,2$ are spinor indices. Consider now the space $\mathcal{P}^{3}=\mathbb{C} P^{3} \backslash \mathbb{C} P^{1}$ in which the $\lambda_{\dot{\alpha}}$ are not simultaneously zero. This space can be covered by two coordinate patches, say $\mathcal{U}_{+}\left(\lambda_{\dot{i}} \neq 0\right)$ and $\mathcal{U}_{-}\left(\lambda_{\dot{2}} \neq 0\right)$, with coordinates

$$
\begin{array}{llll}
z_{+}^{\alpha}=\frac{\omega^{\alpha}}{\lambda_{\dot{1}}}, & z_{+}^{3}=\frac{\lambda_{\dot{2}}}{\lambda_{\dot{1}}}=: \lambda_{+} \quad \text { on } & \mathcal{U}_{+}, \\
z_{-}^{\alpha}=\frac{\omega^{\alpha}}{\lambda_{\dot{2}}}, & z_{-}^{3}=\frac{\lambda_{\dot{i}}}{\lambda_{\dot{2}}}=: \lambda_{-} \quad \text { on } & \mathcal{U}_{-} .
\end{array}
$$

On the intersection $\mathcal{U}_{+} \cap \mathcal{U}_{-}$, they are related by

$$
z_{+}^{\alpha}=\lambda_{+} z_{-}^{\alpha} \quad \text { and } \quad \lambda_{+}=\lambda_{-}^{-1}
$$


It follows from (2.1) and (2.2) that $\mathcal{P}^{3}$ coincides with the total space of the rank 2 holomorphic vector bundle $1 \mathbb{C}^{2} \otimes \mathcal{O}(1)$ over the Riemann sphere $\mathbb{C} P^{1}$. We denote the covering of the latter by $U_{ \pm}=\mathcal{U}_{ \pm} \cap \mathbb{C} P^{1}$ with coordinates $\lambda_{ \pm}$on $U_{ \pm}$.

Real structure. As we are eventually interested in real fields, we need to introduce a real structure on $\mathcal{P}^{3}$. This can be done by defining the anti-linear transformations

$$
\left(\begin{array}{c}
\omega^{1} \\
\omega^{2}
\end{array}\right) \mapsto\left(\begin{array}{c}
-\bar{\omega}^{2} \\
\bar{\omega}^{1}
\end{array}\right) \quad \text { and } \quad\left(\begin{array}{c}
\lambda_{\dot{i}} \\
\lambda_{\dot{2}}
\end{array}\right) \mapsto\left(\begin{array}{c}
-\bar{\lambda}_{\dot{2}} \\
\bar{\lambda}_{\dot{1}}
\end{array}\right)
$$

which induce an antiholomorphic involution (real structure) $\tau$ on $\mathcal{P}^{3}$. On the coordinates (2.1), it acts as follows:

$$
\tau\left(z_{ \pm}^{1}, z_{ \pm}^{2}, z_{ \pm}^{3}\right)=\left( \pm \frac{\bar{z}_{ \pm}^{2}}{\bar{z}_{ \pm}^{3}}, \mp \frac{\bar{z}_{ \pm}^{1}}{\bar{z}_{ \pm}^{3}},-\frac{1}{\bar{z}_{ \pm}^{3}}\right)
$$

It is not difficult to see that $\tau$ has no fixed points in $\mathcal{P}^{3}$ but does leave invariant projective lines $\mathbb{C} P^{1}$ joining the points $p$ and $\tau(p)$ for any $p \in \mathcal{P}^{3}$. For two other possible real structures on $\mathcal{P}^{3}$, see e.g. [14].

Real rational curves. Holomorphic sections of the vector bundle $\mathcal{P}^{3} \rightarrow \mathbb{C} P^{1}$ are rational degree one curves $\mathbb{C} P_{x}^{1} \hookrightarrow \mathcal{P}^{3}$ defined by the equations

$$
z_{ \pm}^{\alpha}=x^{\alpha \dot{\alpha}} \lambda_{\dot{\alpha}}^{ \pm} \quad \text { for } \quad\left(\lambda_{\dot{\alpha}}^{+}\right)=\left(\begin{array}{c}
1 \\
\lambda_{+}
\end{array}\right) \quad \text { and } \quad \lambda_{\dot{\alpha}}^{-}=\lambda_{+}^{-1} \lambda_{\dot{\alpha}}^{+} \quad \text { with } \quad \lambda_{ \pm} \in U_{ \pm}
$$

and parametrized by the moduli $\left(x^{\alpha \dot{\alpha}}\right) \in \mathbb{C}^{4}$. Thus, the complexified spacetime $\mathbb{C}^{4}$ can be identified with the moduli space of holomorphic sections of the bundle $\mathcal{P}^{3} \rightarrow \mathbb{C} P^{1}$.

The action of the map $\tau$ on the $x^{\alpha \dot{\alpha}}$ is given by

$$
\tau\left(\begin{array}{cc}
x^{1 \dot{1}} & x^{1 \dot{2}} \\
x^{2 \dot{1}} & x^{2 \dot{2}}
\end{array}\right)=\left(\begin{array}{cc}
\bar{x}^{2 \dot{2}} & -\bar{x}^{2 \dot{1}} \\
-\bar{x}^{1 \dot{2}} & \bar{x}^{1 \dot{1}}
\end{array}\right)
$$

where the overbar denotes complex conjugation. The real subspace $\mathbb{R}^{4}$ of $\mathbb{C}^{4}$ invariant under $\tau$ is defined by the equations

$$
x^{2 \dot{2}}=\bar{x}^{1 \dot{1}}=: x^{1}-\mathrm{i} x^{2} \quad \text { and } \quad x^{2 \dot{1}}=-\bar{x}^{1 \dot{2}}=: x^{3}-\mathrm{i} x^{4}
$$

and parametrized by the real coordinates $x=\left(x^{\mu}\right) \in \mathbb{R}^{4}$ with $\mu=1, \ldots, 4$. The action of $\tau$ on arbitrary functions $f\left(x, \lambda_{ \pm}, \bar{\lambda}_{ \pm}\right)$is then immediate. Hence, one may impose the

1 Recall that the holomorphic line bundle $\mathcal{O}(m)$ over $\mathbb{C} P^{1}$ is defined by the transition function $\lambda_{+}^{m}$. 
condition of invariance under $\tau$ on sections of the form (2.5) and realize that they are real if the conditions (2.7) hold true.

Metric and signature. On the space $\mathbb{R}^{4}$ of $\tau$-real holomorphic curves $\mathbb{C} P_{x}^{1} \hookrightarrow \mathcal{P}^{3}$, one can introduce the metric

$$
\mathrm{d} s^{2}=\operatorname{det}\left(\mathrm{d} x^{\alpha \dot{\alpha}}\right)=g_{\mu \nu} \mathrm{d} x^{\mu} \mathrm{d} x^{\nu}
$$

which is Euclidean, $\left(g_{\mu \nu}\right)=\operatorname{diag}(+1,+1,+1,+1)$, due to (2.7). Other real structures on $\mathcal{P}^{3}$ produce a metric of signature $(++--)$ on $\mathbb{R}^{4}$ (see e.g. [14]).

Note that topologically, $\mathcal{P}^{3}$ is the direct product

$$
\mathbb{R}^{4} \times \mathbb{C} P^{1} \cong \mathcal{P}^{3}
$$

with coordinates $\left(x^{\mu}, \lambda_{ \pm}\right)$. Therefore, on the patches $\mathcal{U}_{ \pm}$covering $\mathcal{P}^{3}$ one can use either the complex coordinates (2.1) or coordinates $\left(x^{\mu}, \lambda_{ \pm}\right)$. In fact, the formulas (2.5) together with their complex conjugates define a diffeomorphism of $\mathbb{R}^{4} \times \mathbb{C} P^{1}$ onto $\mathcal{P}^{3}$. Its inverse is given by

$$
x^{1 \dot{1}}=\frac{z_{+}^{1}+\bar{z}_{+}^{3} \bar{z}_{+}^{2}}{1+z_{+}^{3} \bar{z}_{+}^{3}}=\frac{\bar{z}_{-}^{3} z_{-}^{1}+\bar{z}_{-}^{2}}{1+z_{-}^{3} \bar{z}_{-}^{3}}, \quad x^{1 \dot{2}}=-\frac{z_{+}^{2}-\bar{z}_{+}^{3} \bar{z}_{+}^{1}}{1+z_{+}^{3} \bar{z}_{+}^{3}}=-\frac{\bar{z}_{-}^{3} z_{-}^{2}-\bar{z}_{-}^{1}}{1+z_{-}^{3} \bar{z}_{-}^{3}},
$$

together with $\lambda_{ \pm}=z_{ \pm}^{3}$ and (2.7) for $x^{2 \dot{2}}$ and $x^{2 \dot{1}}$.

Vector fields. On the complex manifold $\mathcal{P}^{3}$, we have a natural basis $\left(\partial / \partial \bar{z}_{ \pm}^{\alpha}, \partial / \partial \bar{z}_{ \pm}^{3}\right)$ for antiholomorphic vector fields. These are sections of the bundled $\overline{\mathcal{O}}(-1)$ and $\overline{\mathcal{O}}(-2)$, respectively. Using the formulas (2.10), we can express them in terms of the coordinates $\left(x^{\alpha \dot{\alpha}}, \lambda_{ \pm}\right)$according to

$$
\begin{gathered}
\partial_{\bar{z}_{ \pm}^{1}}=-\gamma_{ \pm} \lambda_{ \pm}^{\dot{\alpha}} \partial_{2 \dot{\alpha}}=:-\gamma_{ \pm} \bar{V}_{2}^{ \pm}, \quad \partial_{\bar{z}_{ \pm}^{2}}=\gamma_{ \pm} \lambda_{ \pm}^{\dot{\alpha}} \partial_{1 \dot{\alpha}}=: \gamma_{ \pm} \bar{V}_{1}^{ \pm} \\
\partial_{\bar{z}_{ \pm}^{3}}=\partial_{\bar{\lambda}_{ \pm}}+\gamma_{ \pm} x^{\alpha \dot{1}} \bar{V}_{\alpha}^{ \pm}=: \bar{V}_{3}^{ \pm}+\gamma_{ \pm} x^{\alpha \dot{1}} \bar{V}_{\alpha}^{ \pm}
\end{gathered}
$$

where

$$
\lambda_{ \pm}^{\dot{\alpha}}=\epsilon^{\dot{\alpha} \dot{\beta}} \lambda_{\dot{\beta}}^{ \pm} \quad \text { with } \quad \epsilon^{\dot{1} \dot{2}}=1 \quad \text { and } \quad \gamma_{ \pm}=\left(1+\lambda_{ \pm} \bar{\lambda}_{ \pm}\right)^{-1}
$$

We use $\epsilon_{\dot{\alpha} \dot{\beta}} \epsilon^{\dot{\beta} \dot{\gamma}}=\delta_{\dot{\alpha}}^{\dot{\gamma}}$. Thus, the vector fields

$$
\bar{V}_{\alpha}^{ \pm}=\lambda_{ \pm}^{\dot{\alpha}} \partial_{\alpha \dot{\alpha}} \quad \text { and } \quad \bar{V}_{3}^{ \pm}=\partial_{\bar{\lambda}_{ \pm}}
$$

form a basis of vector fields of type $(0,1)$ on $\mathcal{U}_{ \pm} \subset \mathcal{P}^{3}$ in the coordinates $\left(x^{\alpha \dot{\alpha}}, \lambda_{ \pm}\right)$. For more details on the geometry of the twistor space $\mathcal{P}^{3}$, see e.g. [15] and references therein.

2 Here, $\overline{\mathcal{O}}(m)$ denotes the bundle which is complex conjugate to $\mathcal{O}(m)$. 


\section{Holomorphic Chern-Simons theory on $W \mathbb{C} P^{3 \mid 2}(1,1,1,1 \mid p, q)$}

Weighted projective space $W^{3 \mid 2}(p, q)$. Let us consider the weighted projective space $W^{3 \mid 2}(p, q)=W \mathbb{C} P^{3 \mid 2}(1,1,1,1 \mid p, q)$ with four homogeneous bosonic coordinates $\left(\omega^{\alpha}, \lambda_{\dot{\alpha}}\right)$ of weight one and two fermionic coordinates $\left(\rho_{i}\right)=\left(\rho_{1}, \rho_{2}\right)$ of weight $p$ and $q$, respectively. Here, $\left(\omega^{\alpha}, \lambda_{\dot{\alpha}}\right)$ are the homogeneous coordinates on $\mathbb{C} P^{3}$. Together, the coordinates $\left(\omega^{\alpha}, \lambda_{\dot{\alpha}}, \rho_{i}\right)$ are subject to the equivalence relation $\left(\omega^{\alpha}, \lambda_{\dot{\alpha}}, \rho_{1}, \rho_{2}\right) \sim$ $\left(t \omega^{\alpha}, t \lambda_{\dot{\alpha}}, t^{p} \rho_{1}, t^{q} \rho_{2}\right)$ for all $t \in \mathbb{C}^{*}$. We assume that the sum of fermionic weights is equal to the sum of bosonic weights, i.e. $p+q=4$. In this case, $W^{3 \mid 2}(p, q)$ is a CY supermanifold and one may consider the topological $B$-model of $W^{3 \mid 2}(p, q)$ [困]. The same arguments as given in [1] then yield hCS theory on this space.

Action of hCS theory. Let $\mathcal{E}$ be a trivial rank $n$ complex vector bundle over $W^{3 \mid 2}(p, q)$ and $\mathcal{A}$ a connection one-form on $\mathcal{E}$. Consider a subspace $\mathcal{Y}$ of $W^{3 \mid 2}(p, q)$ which is parametrized by the holomorphic and antiholomorphic bosonic coordinates as well as the holomorphic fermionic coordinates. ${ }^{3}$ The action of hCS theory then reads as [1]

$$
S_{\mathrm{hCS}}=\int_{\mathcal{Y}} \Omega \wedge \operatorname{tr}\left(\mathcal{A}^{0,1} \wedge \bar{\partial} \mathcal{A}^{0,1}+\frac{2}{3} \mathcal{A}^{0,1} \wedge \mathcal{A}^{0,1} \wedge \mathcal{A}^{0,1}\right)
$$

where $\Omega$ is a nowhere vanishing holomorphic volume form and $\mathcal{A}^{0,1}$ denotes the $(0,1)$ component of $\mathcal{A}$. It is assumed that $\mathcal{A}^{0,1}$ contains no antiholomorphic fermionic components and depends only on $\rho_{i}$ as well as on $\left(\omega^{\alpha}, \lambda_{\dot{\alpha}}\right)$ and $\left(\bar{\omega}^{\alpha}, \bar{\lambda}_{\dot{\alpha}}\right)$. The action (3.1) leads to the field equations

$$
\bar{\partial} \mathcal{A}^{0,1}+\mathcal{A}^{0,1} \wedge \mathcal{A}^{0,1}=0 .
$$

Note that for constructing solutions to these equations, one can use a generalization of the Riemann-Hilbert problem based on the equivalence of the Čech and Dolbeault descriptions of holomorphic bundles [16].

Local coordinates. Consider now an open subset of $W^{\left.3\right|^{2}}(p, q)$ defined as $\mathcal{W} \mathcal{P}^{3 \mid 2}(p, q)=W \mathbb{C} P^{3 \mid 2}(1,1,1,1 \mid p, q) \backslash W \mathbb{C} P^{1 \mid 2}(1,1 \mid p, q)$. It can be covered by two patches, which we denote by $\hat{\mathcal{U}}_{ \pm}$. Since the body of the CY supermanifold $\mathcal{W} \mathcal{P}^{3 \mid 2}(p, q)$ is the twistor space $\mathcal{P}^{3}$, we may take the coordinates given in (2.1) as bosonic coordinates on $\hat{\mathcal{U}}_{ \pm}$and as fermionic ones

$$
\rho_{i}^{+}=\frac{\rho_{i}}{\lambda_{\mathrm{i}}} \quad \text { on } \quad \hat{\mathcal{U}}_{+} \quad \text { and } \quad \rho_{i}^{-}=\frac{\rho_{i}}{\lambda_{\dot{2}}} \quad \text { on } \quad \hat{\mathcal{U}}_{-}
$$

which are related by $\rho_{1}^{+}=\lambda_{+}^{p} \rho_{1}^{-}$and $\rho_{2}^{+}=\lambda_{+}^{q} \rho_{2}^{-}$on the intersection $\hat{\mathcal{U}}_{+} \cap \hat{\mathcal{U}}_{-}$. Note that as bosonic coordinates on $\mathcal{W} \mathcal{P}^{3 \mid 2}(p, q)$, we can use either $\left(z_{ \pm}^{\alpha}, z_{ \pm}^{3}\right)$ or $\left(x^{\alpha \dot{\alpha}}, \lambda_{ \pm}\right)$and similarly

3 Recall that $\mathcal{Y}$ is the world-volume of the $D$-branes which are not quite space-filling [1]. 
for the vector fields (2.11) and (2.13). The vector fields 2.13) together with $\partial / \partial \bar{\rho}_{i}$ form a basis of vector fields of type $(0,1)$ on $\mathcal{W P}^{3 \mid 2}(p, q)$.

HCS theory on $\mathcal{W P}^{3 \mid 2}(p, q)$. Having given all the ingredients, we may now consider hCS theory on $\mathcal{W} \mathcal{P}^{3 \mid 2}$ with $p+q=4$. The equations of motion $(3.2)$ on the patches $\hat{\mathcal{U}}_{ \pm}$of $\mathcal{W P}^{3 \mid 2} \mathrm{read}$

$$
\begin{aligned}
\bar{V}_{\alpha}^{ \pm} \mathcal{A}_{\beta}^{ \pm}-\bar{V}_{\beta}^{ \pm} \mathcal{A}_{\alpha}^{ \pm}+\left[\mathcal{A}_{\alpha}^{ \pm}, \mathcal{A}_{\beta}^{ \pm}\right] & =0 \\
\bar{V}_{\alpha}^{ \pm} \mathcal{A}_{\bar{\lambda}_{ \pm}}-\partial_{\bar{\lambda}_{ \pm}} \mathcal{A}_{\alpha}^{ \pm}+\left[\mathcal{A}_{\alpha}^{ \pm}, \mathcal{A}_{\bar{\lambda}_{ \pm}}\right] & =0
\end{aligned}
$$

where

$$
\left.\left.\mathcal{A}_{\alpha}^{ \pm}:=\bar{V}_{\alpha}^{ \pm}\right\lrcorner \mathcal{A}^{0,1} \quad \text { and } \quad \mathcal{A}_{\bar{\lambda}_{ \pm}}:=\partial_{\bar{\lambda}_{ \pm}}\right\lrcorner \mathcal{A}^{0,1}
$$

Here, "」" denotes the interior product of vector fields with differential forms. As usual in the twistor approach, we assume that there exists a gauge in which the components $\mathcal{A}_{\bar{\lambda}_{ \pm}}$ are zero. This corresponds to the holomorphic triviality on any projective line $\mathbb{C} P_{x, \rho}^{1} \hookrightarrow$ $\mathcal{W P}^{3 \mid 2}(p, q)$ of the holomorphic vector bundle which is associated to any solution of hCS theory. From now on, we shall work in the patch $\hat{\mathcal{U}}_{+}$of $\mathcal{W P}^{3 \mid 2}(p, q)$ and for notational simplicity we omit the subscript and the superscript "+" in all expressions except when confusion may arise.

\section{Field models on $\mathbb{R}^{4}$ equivalent to hCS theory on $\mathcal{W} \mathcal{P}^{3 \mid 2}(p, q)$}

HCS theory on $\mathcal{W} \mathcal{P}^{3 \mid 2}(1,3)$. Let us consider the case $p=1$ and $q=3$, where the fermionic coordinates $\rho_{1}$ and $\rho_{2}$ take values in the bundles $\mathcal{O}(1)$ and $\mathcal{O}(3)$, respectively. From (2.13) and (3.5) one concludes that the components $\mathcal{A}_{1}, \mathcal{A}_{2}$ and $\mathcal{A}_{\bar{\lambda}}$ of the $(0,1)$-form $\mathcal{A}^{0,1}$ take values in the bundles $\mathcal{O}(1), \mathcal{O}(1)$ and $\overline{\mathcal{O}}(-2)$. This fixes the dependence of $\mathcal{A}_{\alpha}$ and $\mathcal{A}_{\bar{\lambda}}$ on $\lambda$ and $\bar{\lambda}$ up to gauge transformations. Namely, we obtain

$$
\begin{aligned}
& \mathcal{A}_{\alpha}=\lambda^{\dot{\alpha}} A_{\alpha \dot{\alpha}}+\rho_{1} \chi_{\alpha}+\frac{1}{2 ! \sqrt{3}} \rho_{2} \gamma^{2} \hat{\lambda}^{\dot{\alpha}} \hat{\lambda}^{\dot{\beta}} \widetilde{\chi}_{\alpha \dot{\alpha} \dot{\beta}}+\frac{1}{3 !} \rho_{1} \rho_{2} \gamma^{3} \hat{\lambda}^{\dot{\alpha}} \hat{\lambda}^{\dot{\beta}} \hat{\lambda}^{\dot{\gamma}} G_{\alpha \dot{\alpha} \dot{\beta} \dot{\gamma}} \\
& \mathcal{A}_{\bar{\lambda}}=\frac{1}{\sqrt{3}} \rho_{1} \gamma^{3} \hat{\lambda}^{\dot{\alpha}} \widetilde{\chi}_{\dot{\alpha}}+\frac{1}{2 !} \rho_{1} \rho_{2} \gamma^{4} \hat{\lambda}^{\dot{\alpha}} \hat{\lambda}^{\dot{\beta}} G_{\dot{\alpha} \dot{\beta}}
\end{aligned}
$$

where

$$
\hat{\lambda}^{\dot{\alpha}}:=\tau\left(\lambda^{\dot{\alpha}}\right)=\bar{\lambda}_{\dot{\alpha}}
$$

In (4.1), all the fields $A_{\alpha \dot{\alpha}}, \chi_{\alpha}, \widetilde{\chi}_{\dot{\alpha}}, G_{\dot{\alpha} \dot{\beta}}, \widetilde{\chi}_{\alpha \dot{\alpha} \dot{\beta}}$ and $G_{\alpha \dot{\alpha} \dot{\beta} \dot{\gamma}}$ depend only on $\left(x^{\alpha \dot{\alpha}}\right) \in \mathbb{R}^{4}$.

Substituting (4.1) into (3.4), we get the equations

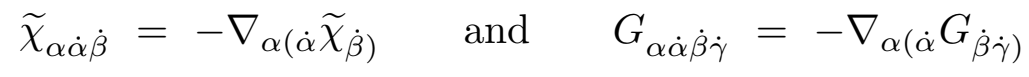


showing that $\widetilde{\chi}_{\alpha \dot{\alpha} \dot{\beta}}$ and $G_{\alpha \dot{\alpha} \dot{\beta} \dot{\gamma}}$ are composite fields describing no independent degrees of freedom. Here, parentheses denote normalized symmetrization with respect to the enclosed indices and $\nabla_{\alpha \dot{\alpha}}=\partial_{\alpha \dot{\alpha}}+\left[A_{\alpha \dot{\alpha}}, \cdot\right]$. The remaining equations are given by

$$
\begin{aligned}
f_{\dot{\alpha} \dot{\beta}}=-\frac{1}{2} \epsilon^{\alpha \beta}\left(\partial_{\alpha \dot{\alpha}} A_{\beta \dot{\beta}}-\partial_{\beta \dot{\beta}} A_{\alpha \dot{\alpha}}+\left[A_{\alpha \dot{\alpha}}, A_{\beta \dot{\beta}}\right]\right) & =0, \\
\nabla_{\alpha \dot{\alpha}} \chi^{\alpha} & =0, \\
\nabla_{\alpha \dot{\alpha}} \tilde{\chi}^{\dot{\alpha}} & =0, \\
\epsilon^{\dot{\alpha} \dot{\beta}} \nabla_{\alpha \dot{\alpha}} G_{\dot{\beta} \dot{\gamma}}-\left\{\chi_{\alpha}, \tilde{\chi}_{\dot{\gamma}}\right\} & =0 .
\end{aligned}
$$

Due to (4.4) and the Bianchi identities, all other equations following from (3.4) are automatically satisfied.

The equations (4.4) are the equations of motion for the action functional

$$
S=\int \mathrm{d}^{4} x \operatorname{tr}\left\{G^{\dot{\alpha} \dot{\beta}} f_{\dot{\alpha} \dot{\beta}}+\widetilde{\chi}^{\dot{\alpha}} \nabla_{\alpha \dot{\alpha}} \chi^{\alpha}\right\}
$$

which can be obtained from (3.1) by integration over the fermionic coordinates and over the sphere $\mathbb{C} P^{1}$. Note that this action has an obvious supersymmetry. The transformation laws are given by

$$
\begin{aligned}
\delta_{\xi} A_{\alpha \dot{\alpha}}=\xi_{\dot{\alpha}} \chi_{\alpha} & \text { and } \quad \delta_{\xi} G_{\dot{\alpha} \dot{\beta}}=-\epsilon^{\alpha \beta} \xi_{(\dot{\alpha}} \nabla_{\alpha \dot{\beta})} \chi_{\beta} \\
\delta_{\xi} \chi_{\alpha}=0 & \text { and } \quad \delta_{\xi} \widetilde{\chi}_{\dot{\alpha}}=-\xi^{\dot{\beta}}\left(G_{\dot{\alpha} \dot{\beta}}+f_{\dot{\alpha} \dot{\beta}}\right)
\end{aligned}
$$

where $\xi_{\dot{\alpha}}$ is a constant (anticommuting) spinor. The action (4.5) describes a truncation of the self-dual $\mathcal{N}=4 \mathrm{SYM}$ model [17] for which all the scalars and three of the dotted and three of the undotted fermions are put to zero.

HCS theory on $\mathcal{W P}^{3 \mid 2}(2,2)$. Now we consider the case $p=q=2$, i.e. the fermionic coordinates $\rho_{i}$ take values in the line bundle $\mathcal{O}(2)$. The equations of motion of hCS theory on $\mathcal{W P}^{3 \mid 2}(2,2)$ have the same form (3.4). Again, the functional dependence on $\lambda$ and $\bar{\lambda}$ is fixed up to gauge transformations by the geometry of $\mathcal{W P}^{3 \mid 2}(2,2)$. Namely, this dependence has the form

$$
\begin{aligned}
& \mathcal{A}_{\alpha}=\lambda^{\dot{\alpha}} A_{\alpha \dot{\alpha}}+\frac{1}{2 !} \rho_{i} \gamma \hat{\lambda}^{\dot{\alpha}} \phi_{\alpha \dot{\alpha}}^{i}+\frac{1}{3 !} \rho_{1} \rho_{2} \gamma^{3} \hat{\lambda}^{\dot{\alpha}} \hat{\lambda}^{\dot{\beta}} \hat{\lambda}^{\dot{\gamma}} G_{\alpha \dot{\alpha} \dot{\beta} \dot{\gamma}} \\
& \mathcal{A}_{\bar{\lambda}}=\frac{1}{2 !} \rho_{i} \gamma^{2} \phi^{i}+\frac{1}{2 !} \rho_{1} \rho_{2} \gamma^{4} \hat{\lambda}^{\dot{\alpha}} \hat{\lambda}^{\dot{\beta}} G_{\dot{\alpha} \dot{\beta}}
\end{aligned}
$$

where again the coefficient fields do only depend on $\left(x^{\alpha \dot{\alpha}}\right) \in \mathbb{R}^{4}$ and $\lambda^{\dot{\alpha}}, \gamma$ and $\hat{\lambda}^{\dot{\alpha}}$ are given in (2.12) and (4.2), respectively. 
Substituting (4.7) into (3.4), we obtain the following equations:

$$
\phi_{\alpha \dot{\alpha}}^{i}=-\nabla_{\alpha \dot{\alpha}} \phi^{i} \quad \text { and } \quad G_{\alpha \dot{\alpha} \dot{\beta} \dot{\gamma}}=-\nabla_{\alpha(\dot{\alpha}} G_{\dot{\beta} \dot{\gamma})} .
$$

The remaining nontrivial equations read

$$
\begin{aligned}
f_{\dot{\alpha} \dot{\beta}} & =0, \\
\nabla_{\alpha \dot{\alpha}} \nabla^{\alpha \dot{\alpha}} \phi^{i} & =0, \\
\epsilon^{\dot{\alpha} \dot{\beta}} \nabla_{\alpha \dot{\alpha}} G_{\dot{\beta} \dot{\gamma}}-\frac{3}{4} \epsilon_{i j}\left\{\phi^{i}, \nabla_{\alpha \dot{\gamma}} \phi^{j}\right\} & =0 .
\end{aligned}
$$

The associated action functional is given by

$$
S=\int \mathrm{d}^{4} x \operatorname{tr}\left\{G^{\dot{\alpha} \dot{\beta}} f_{\dot{\alpha} \dot{\beta}}+\frac{3}{8} \epsilon_{i j} \phi^{i} \nabla_{\alpha \dot{\alpha}} \nabla^{\alpha \dot{\alpha}} \phi^{j}\right\}
$$

and can be obtained from (3.1). Note that formally (4.10) looks as the bosonic truncation of the self-dual $\mathcal{N}=4 \mathrm{SYM}$ theory [17], i.e. all the spinors and four of the six scalars of self-dual $\mathcal{N}=4$ super SYM theory are put to zero. However, in (4.10) the parity of the scalars $\phi^{i}$ is different, as they are Graßmann odd. To understand their nature, note that in the expansions (4.7) we have two Graßmann odd vectors $\phi_{\alpha \dot{\alpha}}^{i}$ which satisfy the equations

$$
\begin{aligned}
\epsilon^{\alpha \beta} \nabla_{\alpha \dot{\alpha}} \phi_{\beta \dot{\beta}}^{i} & =0 \\
\nabla^{\alpha \dot{\alpha}} \phi_{\alpha \dot{\alpha}}^{i} & =0
\end{aligned}
$$

following from (3.4). Solutions to these equations describe tangent vectors $\delta A_{\alpha \dot{\alpha}}$ (with assigned odd parity) to the solution space of the self-duality equations (4.9a) [18, 19. However, due to the first equation in (4.8) (which solves (4.11a) and reduces 4.11b) to $(4.96))$, the $\phi_{\alpha \dot{\alpha}}^{i}$ are projected to zero in the moduli space of solutions to the equations $(4.9 a)$. By choosing $\phi^{i}=0$, we remain with the equations

$$
f_{\dot{\alpha} \dot{\beta}}=0 \quad \text { and } \quad \nabla_{\alpha \dot{\alpha}} G^{\dot{\alpha} \dot{\beta}}=0,
$$

which can be obtained from the Lorentz invariant Siegel action [17

$$
S=\int \mathrm{d}^{4} x \operatorname{tr}\left\{G^{\dot{\alpha} \dot{\beta}} f_{\dot{\alpha} \dot{\beta}}\right\}
$$

describing purely bosonic self-dual Yang-Mills theory.

HCS theory on $\mathcal{W P}^{3 \mid 2}(4,0)$. Finally, we want to discuss the case in which the fermionic coordinate $\rho_{1}$ has weight four and $\rho_{2}$ weight zero, i.e. we consider $\mathcal{W P}^{3 \mid 2}(4,0)$. The field equations of hCS theory on $\mathcal{W P}^{3 \mid 2}(4,0)$ have the form (3.4), where the vector 
fields $\bar{V}_{\alpha}^{ \pm}$and $\partial_{\bar{\lambda}_{ \pm}}$are given in (2.13). Proceeding as in the previous two subsections, we obtain the following field expansions:

$$
\begin{aligned}
& \mathcal{A}_{\alpha}=\lambda^{\dot{\alpha}} A_{\alpha \dot{\alpha}}+\frac{1}{3 !} \rho_{1} \gamma^{3} \hat{\lambda}^{\dot{\alpha}} \hat{\lambda}^{\dot{\beta}} \hat{\lambda}^{\dot{\gamma}} \chi_{\alpha \dot{\alpha} \dot{\beta} \dot{\gamma}}+\rho_{2} \lambda^{\dot{\alpha}} \psi_{\alpha \dot{\alpha}}+\frac{1}{3 !} \rho_{1} \rho_{2} \gamma^{3} \hat{\lambda}^{\dot{\alpha}} \hat{\lambda}^{\dot{\beta}} \hat{\lambda}^{\dot{\gamma}} G_{\alpha \dot{\alpha} \dot{\beta} \dot{\gamma}} \\
& \mathcal{A}_{\bar{\lambda}}=\frac{1}{2 !} \rho_{1} \gamma^{4} \hat{\lambda}^{\dot{\alpha}} \hat{\lambda}^{\dot{\beta}} \chi_{\dot{\alpha} \dot{\beta}}+\frac{1}{2 !} \rho_{1} \rho_{2} \gamma^{4} \hat{\lambda}^{\dot{\alpha}} \hat{\lambda}^{\dot{\beta}} G_{\dot{\alpha} \dot{\beta}} .
\end{aligned}
$$

All the Lie-algebra valued fields appearing in the expansions (4.14) depend only on $\left(x^{\alpha \dot{\alpha}}\right) \in$ $\mathbb{R}^{4}$. Note also that $A_{\alpha \dot{\alpha}}, G_{\dot{\alpha} \dot{\beta}}$ and $G_{\alpha \dot{\alpha} \dot{\beta} \dot{\gamma}}$ are bosonic while $\psi_{\alpha \dot{\alpha}}, \chi_{\dot{\alpha} \dot{\beta}}$ and $\chi_{\alpha \dot{\alpha} \dot{\beta} \dot{\gamma}}$ are fermionic fields.

Substituting (4.14) into (3.4), we obtain

$$
\chi_{\alpha \dot{\alpha} \dot{\beta} \dot{\gamma}}=-\nabla_{\alpha(\dot{\alpha}} \chi_{\dot{\beta} \dot{\gamma})} \quad \text { and } \quad G_{\alpha \dot{\alpha} \dot{\beta} \dot{\gamma}}=-\nabla_{\alpha(\dot{\alpha}} G_{\dot{\beta} \dot{\gamma})}-\left\{\psi_{\alpha(\dot{\alpha}}, \chi_{\dot{\beta} \dot{\gamma})}\right\}
$$

The remaining nontrivial equations are

$$
\begin{aligned}
f_{\dot{\alpha} \dot{\beta}} & =0, \\
\epsilon^{\alpha \beta} \nabla_{\alpha \dot{\alpha}} \psi_{\beta \dot{\beta}} & =0, \\
\nabla_{\alpha \dot{\alpha}} G^{\dot{\alpha} \dot{\beta}}+\left\{\psi_{\alpha \dot{\alpha}}, \chi^{\dot{\alpha} \dot{\beta}}\right\} & =0, \\
\nabla_{\alpha \dot{\alpha}} \chi^{\dot{\alpha} \dot{\beta}} & =0 .
\end{aligned}
$$

In this case, the action functional from which these equations arise is

$$
S=\int \mathrm{d}^{4} x \operatorname{tr}\left\{G^{\dot{\alpha} \dot{\beta}} f_{\dot{\alpha} \dot{\beta}}+\epsilon^{\alpha \beta}\left(\nabla_{\alpha \dot{\alpha}} \psi_{\beta \dot{\beta}}\right) \chi^{\dot{\alpha} \dot{\beta}}\right\}
$$

This time, the multiplet contains a spacetime vector $\psi_{\alpha \dot{\alpha}}$ and an anti-self-dual two-form $\chi_{\dot{\alpha} \dot{\beta}}$ which are both Graßmann odd. Such fields are well known from topological Yang-Mills theories [18,19]. In this respect, the model (4.16), (4.17) can be understood as a truncated self-dual sector of these theories.4

\section{Acknowledgments}

We thank Olaf Lechtenfeld, Christian Sämann and Robert Wimmer for useful comments. This work was partially supported by the Deutsche Forschungsgemeinschaft (DFG).

4 One may also consider more than two fermionic coordinates in order to enlarge the multiplet. This may lead to other truncations of topological Yang-Mills theories. 


\section{Appendix A. Signature $(++--)$}

For the Kleinian space $\mathbb{R}^{2,2}=\left(\mathbb{R}^{4}, g\right)$ with the metric $g=\operatorname{diag}(+1,+1,-1,-1)$, all the calculations and formulas are similar. What should be changed is the following. Instead of the reality conditions (2.7), one considers

$$
x^{2 \dot{2}}=\bar{x}^{1 \dot{1}}=: x^{1}-\mathrm{i} x^{2} \quad \text { and } \quad x^{2 \dot{1}}=\bar{x}^{1 \dot{2}}=: x^{3}+\mathrm{i} x^{4}
$$

which correspond to the involution (real structure)

$$
\hat{\tau}\left(z_{ \pm}^{1}, z_{ \pm}^{2}, z_{ \pm}^{3}\right)=\left(\frac{\bar{z}_{ \pm}^{2}}{\bar{z}_{ \pm}^{3}}, \frac{\bar{z}_{ \pm}^{1}}{\bar{z}_{ \pm}^{3}}, \frac{1}{\bar{z}_{ \pm}^{3}}\right)
$$

on the twistor space $\mathcal{P}^{3}$. Furthermore, instead of $\mathcal{P}^{3}$ one should consider the spact $\widetilde{\mathcal{P}}^{3}=\mathcal{P}^{3} \backslash \mathcal{T}^{3}$, where $\mathcal{T}^{3}$ is the fixed point set under the involution $\hat{\tau}$. In fact, $\mathcal{T}^{3}$ is the three-dimensional real manifold $\mathbb{R} P^{3} \backslash \mathbb{R} P^{1}$ fibered over $S^{1} \cong \mathbb{R} P^{1} \subset \mathbb{C} P^{1}$. All CY supermanifolds $\mathcal{W} \mathcal{P}^{3 \mid 2}(p, q)$ considered above are now supermanifolds with $\widetilde{\mathcal{P}}^{3}$ as body. One should also substitute $\mathbb{C} P^{1}$ by the two-sheeted hyperboloid $H^{2}=\mathbb{C} P^{1} \backslash S^{1}=H_{+} \cup H_{-}$ and use

$$
\left(\hat{\lambda}_{+}^{\dot{\alpha}}\right)=\left(\begin{array}{c}
-1 \\
\bar{\lambda}_{+}
\end{array}\right), \quad\left(\hat{\lambda}_{-}^{\dot{\alpha}}\right)=\left(\begin{array}{c}
-\bar{\lambda}_{-} \\
1
\end{array}\right), \quad \gamma_{ \pm}= \pm\left(1-\lambda_{+} \bar{\lambda}_{+}\right)^{-1} \quad \text { with } \quad \lambda_{ \pm} \in H_{ \pm}
$$

instead of $\gamma_{ \pm}$and $\hat{\lambda}_{ \pm}^{\dot{\alpha}}$ as given in (2.12) and (4.2), respectively. All other formulas, including the equations of motion for hCS theory and the field expansions of $\mathcal{A}^{0,1}$ keep their form. The resulting field equations on $\mathbb{R}^{2,2}$ will only differ by some signs in front of the interaction terms.

5 For more details see e.g. 20]. 


\section{References}

[1] E. Witten, Perturbative gauge theory as a string theory in twistor space, hepth/0312171.

[2] E. Witten, Chern-Simons gauge theory as a string theory, Prog. Math. 133 (1995) 637 hep-th/9207094.

[3] N. Berkovits, An alternative string theory in twistor space for $\mathcal{N}=4$ super-YangMills, hep-th/0402045; Self-dual super-Yang-Mills as a string theory in $(x, \theta)$-space, hep-th/0403280.

[4] A. Neitzke and C. Vafa, $N=2$ strings and the twistorial Calabi-Yau, hep-th/0402128;

N. Nekrasov, H. Ooguri and C. Vafa, S-duality and topological strings, hep-th/0403167.

[5] M. Aganagic and C. Vafa, Mirror symmetry and supermanifolds, hep-th/0403192; S. P. Kumar and G. Policastro, Strings in twistor superspace and mirror symmetry, hep-th/0405236.

[6] N. Berkovits and L. Motl, Cubic twistorial string field theory, JHEP 0404 (2004) 056 hep-th/0403187.

[7] W. Siegel, Untwisting the twistor superstring, hep-th/0404255.

[8] N. Berkovits and E. Witten, Conformal supergravity in twistor-string theory, hepth/0406051.

[9] O. Lechtenfeld and A. D. Popov, Supertwistors and cubic string field theory for open $N=2$ strings, hep-th/0406179.

[10] V. P. Nair, A current algebra for some gauge theory amplitudes, Phys. Lett. B 214 (1988) 215.

[11] R. Roiban, M. Spradlin and A. Volovich, A googly amplitude from the $B$-model in twistor space, JHEP 0404 (2004) 012 hep-th/0402016; On the tree-level S-matrix of Yang-Mills theory, hep-th/0403190; R. Roiban and A. Volovich, All googly amplitudes from the B-model in twistor space, hep-th/0402121; G. Georgiou and V. V. Khoze, Tree amplitudes in gauge theory as scalar MHV diagrams, JHEP 0405 (2004) 070 hep-th/0404072.

[12] F. Cachazo, P. Svrcek and E. Witten, MHV vertices and tree amplitudes in gauge theory, hep-th/0403047; Twistor space structure of one-loop amplitudes in gauge theory, hep-th/0406177; C. J. Zhu, The googly amplitudes in gauge theory, JHEP 0404 (2004) 032 [hep-th/0403115; J. B. Wu and C. J. Zhu, MHV vertices and scattering amplitudes in gauge theory, hep-th/0406085; $M H V$ vertices and fermionic scattering amplitudes in gauge theory with quarks and gluinos, hep-th/0406146.

[13] E. Witten, Parity invariance for strings in twistor space, hep-th/0403199; S. Gukov, L. Motl and A. Neitzke, Equivalence of twistor prescriptions for super Yang-Mills, hep-th/0404085. 
[14] A. D. Popov and C. Sämann, On supertwistors, the Penrose-Ward transform and $\mathcal{N}=4$ super Yang-Mills theory, hep-th/0405123.

[15] A. D. Popov, Self-dual Yang-Mills: Symmetries and moduli space, Rev. Math. Phys. 11 (1999) 1091 hep-th/9803183.

[16] A. D. Popov, Holomorphic Chern-Simons-Witten theory: From 2D to $4 D$ conformal field theories, Nucl. Phys. B 550 (1999) 585 hep-th/9806239; T. A. Ivanova and A. D. Popov, Dressing symmetries of holomorphic BF theories, J. Math. Phys. 41 (2000) 2604 hep-th/0002120.

[17] W. Siegel, Phys. Rev. D 46 (1992) R3235 hep-th/9205075; G. Chalmers and W. Siegel, The self-dual sector of QCD amplitudes, Phys. Rev. D 54 (1996) 7628 hep-th/9606061.

[18] E. Witten, Topological quantum field theory, Commun. Math. Phys. 117 (1988) 353.

[19] C. Vafa and E. Witten, A Strong coupling test of S-duality, Nucl. Phys. B 431 (1994) 3 hep-th/9408074.

[20] O. Lechtenfeld and A. D. Popov, Closed $N=2$ strings: Picture-changing, hidden symmetries and SDG hierarchy, Int. J. Mod. Phys. A 15 (2000) 4191 [hep-th/9912154]; T. A. Ivanova and O. Lechtenfeld, Hidden symmetries of the open $N=2$ string, Int. J. Mod. Phys. A 16 (2001) 303 [hep-th/0007049]. 